\title{
Comprehensive, multidimensional and correlative particle characterization of a saxolite and talcum compound to support the understanding of complex separation processes
}

Silvan Englisch ${ }^{1}$, Ralf Ditscherlein ${ }^{2}$, Orkun Furat ${ }^{3}$, Leonard Hansen ${ }^{4}$, Dominik Drobek ${ }^{5}$, Janis Wirth ${ }^{1}$, Simon Carl ${ }^{1}$, Thomas Leißner ${ }^{2}$, Benjamin Apeleo Zubiri ${ }^{1}$, Alfred Weber ${ }^{4}$, Volker Schmidt ${ }^{3}$, Urs Peuker ${ }^{2}$ and Erdmann Spiecker ${ }^{1}$

${ }^{1}$ Institute of Micro- and Nanostructure Research (IMN) \& Center for Nanoanalysis and Electron Microscopy (CENEM), Interdisciplinary Center for Nanostructured Films (IZNF), Department of Materials Science and Engineering, Friedrich-Alexander University Erlangen-Nürnberg (FAU), Erlangen, Germany, Erlangen, Bayern, Germany, ${ }^{2}$ Institute of Mechanical Process Engineering and Mineral Processing, Technical University Bergakademie Freiberg, Germany, United States, ${ }^{3}$ Institute of Stochastics, Ulm University, Germany, United States, ${ }^{4}$ Institute of Particle Technology, Clausthal University of Technology, Germany, United States, ${ }^{5}$ Institute of Micro- and Nanostructure Research (IMN) \& Center for Nanoanalysis and Electron Microscopy (CENEM), Interdisciplinary Center for Nanostructured Films (IZNF), Department of Materials Science and Engineering, Friedrich-Alexander University Erlangen-Nürnberg (FAU), Erlangen, Germany, United States

We present a correlative 3D characterization workflow by micro X-ray computed tomography (Micro$\mathrm{CT}$ ), nano X-ray computed tomography (Nano-CT) and analytical scanning electron microscopy (SEM) over different length scales for a particle composite material featuring two morphologically similar particulate minerals - saxolite and talcum. The results of this comprehensive 3D microscopy study serve as a reference to support the understanding of complex separation processes and the optimization of multidimensional separation efficiency achieved by a combination of a deflector wheel classifier and an integrated electric sorter, using the triboelectric charge [1]. This combination separates mineral powders according to their morphology, size distribution, and chemical composition of each particle fraction. Evaluating the particle properties with complementary characterization techniques before and, in particular, after the separation process is critical to determine the multidimensional sorting efficiency.

In this case study, an artificial compound of saxolite and talcum (talcum contains foremostly the minerals: talcum, dolomite, and magnesite) provides constituent particles with similar morphology and size distribution as well as partly similar chemical composition, emulating a snapshot before the separation process. For the microscopic characterization workflow, the different minerals with unequal ratios are embedded in epoxy resin mixed with graphite nanoparticles to avoid sample movement during characterization and to space out the particles to facilitate image segmentation.

The 3D characterization workflow, depicted in Figure 1, consists of the following steps: First, a sample with a volume of $(400 \mu \mathrm{m})^{3}$ is prepared from the composite material manually by scalpel cutting for Micro-CT analysis [2]. Absorption contrast imaging in a lab-based Zeiss Xradia 510 Versa Micro-CT instrument reveals the 3D morphology and distribution of different particles and cavities in the pillar volume with a resolution of $(400 \mathrm{~nm})^{3}$ (Figure 1 Micro-CT). Secondly, for a correlative Nano-CT investigation at higher resolution, a representative region of interest (ROI) is site-specifically prepared by cutting a free-standing pillar of $200 \mu \mathrm{m}$ in height and $60 \mu \mathrm{m}$ in diameter out of the bigger pillar with a 3D-Micromac microPREP PRO pico-second laser ablation system. To enable the 3D reconstruction of the entire pillar volume, three vertically adjacent absorption contrast large-field-of-view (LFOV) Nano-CT 
tilt series are acquired with a Zeiss Xradia 810 Ultra Nano-CT instrument. The lab-based X-ray microscope is equipped with a $5.4 \mathrm{keV} \mathrm{K}_{\alpha}$ rotating Chromium-anode source and allows for imaging with quasi-monochromatic X-rays covering a field of view of $65 \mu \mathrm{m}$ in a single tilt series with a spatial resolution of $(150 \mathrm{~nm})^{3}$. In absorption contrast mode, the attenuation of the transmitted X-rays is governed by the Beer-Lambert law, so the higher the atomic number of the sample constituent and the higher the sample thickness, the lower the transmitted X-ray signal (Figure 1 Nano-CT). The inherent mass-thickness contrast facilitates to distinguish particles with significantly different densities or chemical compositions. In a third characterization step, the Nano-CT pillar is analyzed with the slice \& view method in a DualBeam FEI Helios NanoLab 660 focused ion beam (FIB)-SEM instrument. Here, sufficiently precise local chemical information is obtained by recording backscattered electron (BSE) images and energy-dispersive X-ray spectroscopy (EDXS) maps of only a few slices in each of the three corresponding Nano-CT volumes instead of imaging all possible slices (Figure 1 FIB-SEM).

The imaged FIB-SEM slices are directly correlated with corresponding slices in the Nano-CT reconstructions and enable identifying the chemical composition of all particles in the 3D volume. Each expected mineral type can be unswervingly assigned to the particles by applying this approach. All particles from a respective mineral type are segmented in Arivis Vision4D by applying a machine-learning algorithm [3] and classified regarding their specific morphology, frequency and distribution, starting with the BSE/EDXS-informed Nano-CT virtual slices, according to Lenz, Wirth et al. [4]. The segmentation routine transfers the chemical information to the entire Nano-CT volume, providing each mineral's morphology and distribution. Finally, the routine is continued with the segmentation of the Micro-CT volume, where the Nano-CT analysis informs the segmentation algorithm of the corresponding ROI in the Micro-CT reconstruction. The final statistical interpretation is improved by advanced post-processing and multidimensional stochastic modeling, according to Furat et al. [5]. This workflow provides multidimensional probability distributions of particle descriptors for the size, shape and chemical composition - allowing for a quantitative comparison between measured compounds of the educt and product of the considered multidimensional separation process.

In summary, the presented characterization workflow over different length scales is utilized to unambiguously allocate and identify the mineral type of similar-sized particles in a large volume by a comprehensive evaluation of the particles' morphology, distribution and composition. The combination of several methods significantly improves the statistical relevance (high number of particles) and segmentation precision (high resolution) compared to a single technique. The gathered microscopic data provides a point of reference concerning the particle properties for the multidimensional separation efficiency achieved by a combination of deflector wheel classifier and triboelectric charge sorting. Additionally, it can be used as an input for modeling and simulation to obtain a detailed understanding of these complex separation processes [6]. 


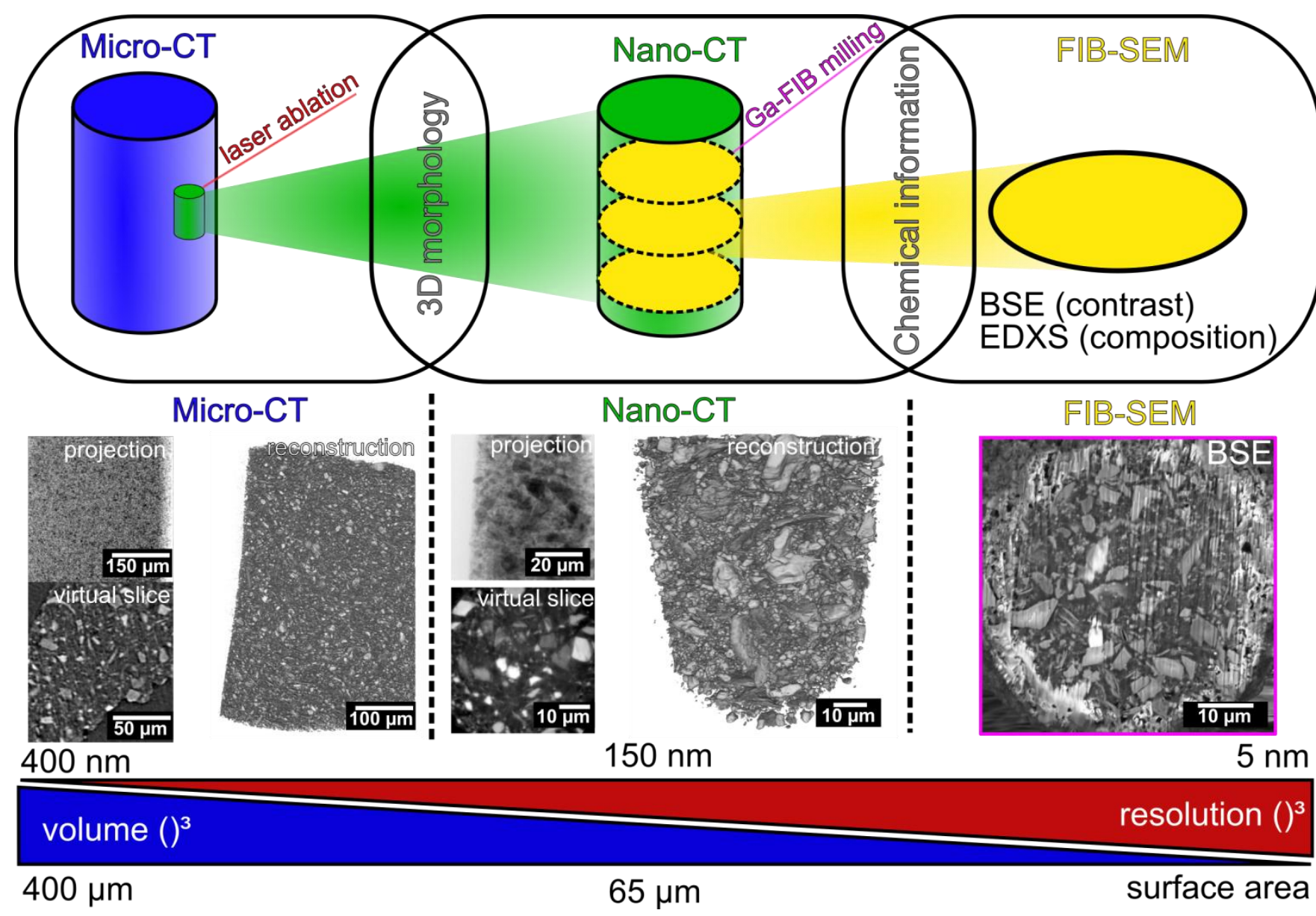

Figure 1. Correlative Workflow: In a first step, a volume of $(400 \mu \mathrm{m})^{3}$ is prepared manually to enable Micro-CT imaging with a resolution of $(400 \mathrm{~nm})^{3}$. In the second step, a region of interest is cut out sitespecifically by a pico-second laser cutter for Nano-CT exploration at a higher spatial resolution of (150 $\mathrm{nm})^{3}$. The resulting pillar with a diameter of $65 \mu \mathrm{m}$ is sliced and imaged by the FIB-SEM system in the third step. Micro- and Nano-CT examine the 3D morphology of the particles included in the composite. The chemical information is provided by the attenuation lengths of the different materials in the absorption contrast Nano-CT reconstruction and BSE contrast in the SEM images (corresponding gray values in virtual slice and BSE image). In addition, EDXS of the prepared slices determines the exact chemical composition (cf. Figure 2). The mineral information gathered at high-resolution by BSE/EDXS FIB-SEM informs first the segmentation of the Nano-CT volume and further of the Micro-CT volume.
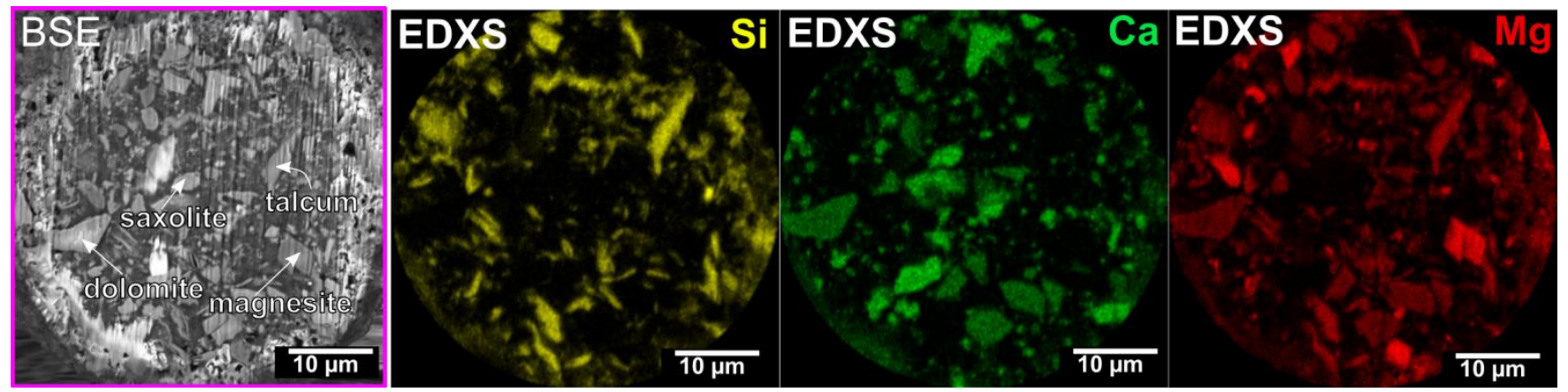

Figure 2. Allocation of the mixed minerals in a Nano-CT pillar: (a) EDXS analysis of the site-specifically prepared slices after FIB milling and polishing. Each mineral in the composition is assigned by detecting 
the constituent elements silicon, calcium and magnesium, for instance, saxolite (main constituent element $\mathrm{Ca})$, talcum $(\mathrm{Si}, \mathrm{Mg})$, dolomite $(\mathrm{Ca}, \mathrm{Mg})$ and magnesite $(\mathrm{Mg})$. This chemical allocation corroborates the estimated grayscale for the minerals in the BSE images and the Nano-CT reconstruction and informs the segmentation of the Nano-CT volume.

\section{References}

1. Hansen, L., Wollmann, A., Weers, M., Benker, B. and Weber, A.P. (2020), Triboelectric charging and separation of fine powder mixtures. Chem. Eng. Technol., 43: 933-941.

2. Ralf Ditscherlein, Thomas Leißner, Urs A. Peuker, (2020), Preparation techniques for micron-sized particulate samples in X-ray microtomography, Powder Technology, 360: 989-997,ISSN 0032-5910

3. C. Sommer, C. Straehle, U. Köthe, F. A. Hamprecht (2016),, Ilastik: interactive learning and segmentation toolkit. IEEE International Symposium on Biomedical Imaging: From Nano to Macro, 2011. 4. Lenz M., Wirth J., Englisch S., Rosiwal J., Buchinger N., Weiser M., Virtanen S., Apeleo Zubiri B., Spiecker E.: (2019), Correlative nano-computed tomography and focused ion-beam sectioning: A case study on a Co-base superalloy oxide scale. In: Advanced Engineering Materials, Article No.: 1900823 ISSN: $1438-1656$

5. Furat, O., Leißner, T., Bachmann, K., Gutzmer, J., Peuker, U.A. and Schmidt, V. (2019), Stochastic modeling of multidimensional particle characteristics using parametric copulas. Microscopy and Microanalysis, 25:720-734.

We gratefully acknowledge financial support by the German Research Foundation (DFG) within the frameworks of the research training group GRK1896 "In situ Microscopy with Electrons, X-rays and Scanning Probes", the project SP648/8 "High-resolution X-ray microscopy for correlative tomography, high throughput screening and in situ mechanical testing of structural and functional materials" (ProjectID 316992193), the Collaborative Research Centre 1411 "Design of Particulate Products" (Project-ID 416229255), the Collaborative Research Centre 1452 "Catalysis at Liquid Interfaces", the project INST 267/129-1 and the Priority Program SPP2045 "(DFG 313858373). 